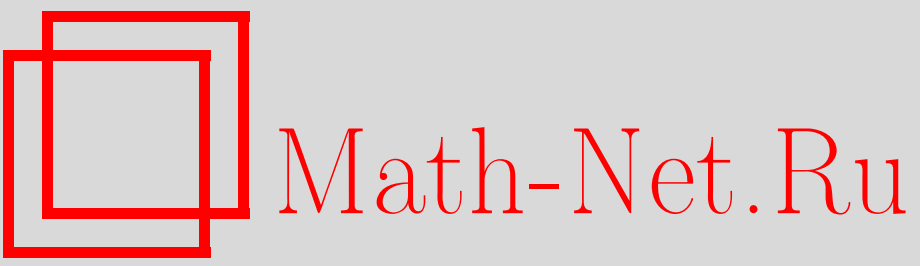

Б. М. Барбашов, А. Б. Пестов, Антисимметричные тензорные поля и калибровочная теория Вейля, ТМФ, 1997, том 113, номер 1, 112-123

DOI: https://doi.org/10.4213/tmf1069

Использование Общероссийского математического портала Math-Net.Ru подразумевает, что вы прочитали и согласны с пользовательским соглашением

http://www.mathnet.ru/rus/agreement

Параметры загрузки:

IP: 54.237 .206 .68

26 апреля 2023 г., 17:31:26 


\author{
ТЕОРЕТИЧЕСКАЯ \\ И МАТЕМАТИЧЕСКАЯ \\ ФИЗИКА \\ Том 113, № 1 \\ октябрь, 1997
}

Б. М. Барбашов* , А.Б. Пестов*

\title{
АНТИСИММЕТРИЧНЫЕ ТЕНЗОРНЫЕ ПОЛЯ И КАЛИБРОВОЧНАЯ ТЕОРИЯ ВЕЙЛЯ
}

Цель работы - найти спинорный ток - источник в неабелевой калибровочной теории Вейля, которая характерна тем, что в ней нет абстрактного калибровочного пространства. Показано, что искомое спинорное представление калибровочной группы Вейля может быть построено в пространстве антисимметричных тензорных полей и является 16 -компонентной величиной, для которой установлен калибровочно-инвариантный лагранжиан. Обсуждаются связь между неабелевым калибровочным потенциалом Вейля и полем кручения Картана и вопрос о том, где могут проявляться рассмотренные взаимодействия.

\section{1. ВВЕДЕНИЕ}

В работе [1] было показано, что конгруэнтный перенос, введенный в 1921 г. Вейлем [2], определяет неабелево калибровочное поле. Сформулированная в [1] калибровочная теория Вейля является такой реализацией абстрактной теории калибровочных полей в рамках классической дифференциальной геометрии, в которой отсутствует разделение между пространством-временем и калибровочным пространством, в то время как стандартные калибровочные модели взаимодействий предполагают точное локальное разделение между пространством-временем и калибровочным пространством. Именно в этом пункте калибровочная теория Вейля открьвает новую возможность.

Здесь показано, что пространство всех ковариантных антисимметричных тензорных полей является спинорным представлением калибровочной группы Вейля и дает возможность построить спинорный ток - источник в такой калибровочной теории. Рассмотрен статус тензора кручения Картана в рамках калибровочной теории Вейля, и показано, что в определенной калибровке (которая выводится) калибровочно-инвариантные уравнения поля могут быть записаны как уравнения в пространстве Римана-Картана.

\section{2. КАЛИБРОВОЧНЫЙ ПОТЕНЦИАЛ}

Связность Вейля, определяюшую конгруэнтный перенос вектора, запишем в виде

$$
\Gamma_{j k}^{i}=\left\{\begin{array}{l}
i \\
j k
\end{array}\right\}+G_{j k}^{i},
$$

* Объединенный институт ядерных исследований, Дубна, Московская обл., Россия. E-mail: pestov@theor.jinr.dubna.su 
где $\left\{\begin{array}{c}i \\ j k\end{array}\right\}$ - символы Кристоффеля римановой связности метрики $g_{i j}$ :

$$
\left\{\begin{array}{l}
i \\
j k
\end{array}\right\}=\frac{1}{2} g^{i l}\left(\partial_{j} g_{k l}+\partial_{k} g_{j l}-\partial_{l} g_{j k}\right)
$$

a $G_{j k}^{i}=G_{j k l} g^{i l}$ - компоненты неабелева калибровочного потенциала Вейля, который является ковариантным тензором третьего ранга, кососимметричным по двум последним индексам,

$$
G_{j k l}+G_{j l k}=0 .
$$

Согласно (1) компоненты вектора при конгруэнтном переносе изменяются по закону

$$
d v^{i}=-\left\{\begin{array}{c}
i \\
j k
\end{array}\right\} d x^{j} v^{k}-G_{j k}^{i} d x^{j} v^{k},
$$

который включает в себя смешение, принадлежашее римановой геометрии, и врашение, определяемое метрикой $g_{i j}$ и бивектором $G_{j k l} d x^{j}$. Обозначим через $\stackrel{*}{\nabla}_{i}$ ковариантную производную относительно связности (1). Тогда с учетом (3) получаем

$$
\stackrel{\nabla}{\nabla}_{i} g_{j k}=0 \text {. }
$$

Таким образом, связность Вейля является метрической.

Представленная геометрическая конструкция Вейля имеет простой теоретико-групповой смысл. Пусть $S_{j}^{i}$ - компоненты тензорного поля $S$ типа $(1,1)$, удовлетворяющего условию $\operatorname{det}\left(S_{j}^{i}\right) \neq 0$. В этом случае сушествует тензорное поле $S^{-1}$ с компонентами $T_{j}^{i}$ такое, что

$$
S_{k}^{i} T_{j}^{k}=\delta_{j}^{i}
$$

Очевидно, что тензорное поле $S$ задает линейное преобразование

$$
\bar{v}^{i}=S_{j}^{i} v^{j}
$$

в пространстве векторных полей; $S^{-1}$ является обратным преобразованием. В последующем будут рассматриваться только такие преобразования (6), которые не изменяют длину вектора. Это дополнительное условие задается уравнениями

$$
g_{i k} S_{j}^{k}=g_{j k} T_{i}^{k}
$$

Преобразования вида (6) и (7) образуют группу, которая, как будет показано, является калибровочной группой. Обозначим ее $G_{W}$. Таким образом, формулы $(6)$ и $(7)$ задают отношение эквивалентности в пространстве векторных полей. Классы эквивалентности отличаются длиной входящих в них векторов. В последующем будут указаны другие важные представления калибровочной группы $G_{W}$.

Можно показать, что если какой-либо вектор $v^{i}$ из класса эквивалентности испытывает конгруэнтный перенос, то и любой эквивалентный ему в смысле группы $G_{W}$ вектор $\bar{v}^{i}=S_{j}^{i} v^{j}$ также испытывает конгруэнтный перенос. Другими словами, если для $v^{i}$ имеет место (4), то для $\bar{v}^{i}=S_{j}^{i} v^{j}$ справедливо соотношение

$$
d \bar{v}^{i}=-\left\{\begin{array}{l}
i \\
j k
\end{array}\right\} d x^{j} \bar{v}^{k}-\bar{G}_{j k}^{i} d x^{j} \bar{v}^{k},
$$


где

$$
\bar{G}_{l k m}=G_{l i j} T_{k}^{i} T_{m}^{j}+g_{i j} T_{k}^{i} \nabla_{l} T_{m}^{j} .
$$

В формуле (8) и в дальнейшем $\nabla_{i}$ обозначает ковариантную производную по отношению к римановой связности (2). Из (7) следует, что тензор $\bar{G}_{l k m}$ удовлетворяет уравнению (3), и, следовательно, связность Вейля

$$
\bar{\Gamma}_{j k}^{i}=\left\{\begin{array}{c}
i \\
j k
\end{array}\right\}+\bar{G}_{j k}^{i}
$$

также задает конгруэнтный перенос. Таким образом, наряду с данной метрической связностью мы имеем целый класс эквивалентных метрических связностей. Вместе с тем мы построили нелинейное представление рассматриваемой калибровочной групшы.

Рассмотрим бесконечно малое калибровочное преобразование $S_{j}^{i}=\delta_{j}^{i}+u_{j}^{i}, T_{j}^{i}=$ $\delta_{j}^{i}-u_{j}^{i}$, которое после подстановки в (7) дает $g_{i k} u_{j}^{k}+g_{j k} u_{i}^{k}=0$. Отсюда следует, что всякое антисимметричное ковариантное тензорное поле второго ранга (2-форма) $u_{i j}=$ $-u_{j i}$ задает бесконечно малое калибровочное преобразование, так как $u_{j}^{i}=u_{j k} g^{i k}$. Коммутатор двух преобразований вида $\bar{v}^{i}=u_{j}^{i} v^{j}$ снова является преобразованием такого же вида, так как

$$
w_{i j}=u_{i m} v_{j}^{m}-u_{j m} v_{i}^{m} .
$$

Из (8) получаем, что бесконечно малое калибровочное преобразование потенциала имеет вид

$$
\delta G_{i j k}=\nabla_{i} u_{j k}+G_{i j l} u_{k}^{l}-G_{i k l} u_{j}^{l} .
$$

Покажем теперь, что тензор напряженности калибровочного поля совпадает с тензором кривизны связности (1). Тензор кривизны связности (1) может быть записан в виде

$$
B_{i j k l}=\nabla_{i} G_{j k l}-\nabla_{j} G_{i k l}+G_{i k m} G_{j l}^{m}-G_{j k m} G_{i l}^{m}+R_{i j k l},
$$

где $R_{i j k l}$ - тензор римановой кривизны метрики $g_{i j}$. Положим $B_{i j k l}=H_{i j k l}+R_{i j k l}$. Из (9), (10) получаем

$$
\bar{H}_{i j k l}=H_{i j k l}+H_{i j k m} u_{l}^{m}-H_{i j l m} u_{k}^{m}+\left(\nabla_{i} \nabla_{j}-\nabla_{j} \nabla_{i}\right) u_{k l} .
$$

Согласно тождествам Риччи коммутатор ковариантных производных равен

$$
\left(\nabla_{i} \nabla_{j}-\nabla_{j} \nabla_{i}\right) u_{k l}=R_{i j k m} u_{l}^{m}-R_{i j l m} u_{k}^{m} .
$$

Отсюда следует, что тензор $B_{i j k l}$ преобразуется при калибровках по закону

$$
\delta B_{i j k l}=B_{i j k m} u_{l}^{m}-B_{i j l m} u_{k}^{m}
$$

и, следовательно, является тензором напряженности калибровочного поля.

Таким образом, тензорное поле $G_{i j k}$, входящее в связность Вейля, является калибровочным полем, а тензор $B_{i j k l}$ - тензор напряженности этого поля. Подчеркнем, что в рассматриваемом случае калибровочная группа определяется метрикой, а калибровочное поле имеет прямой геометрический смысл (конгруэнтный перенос), при этом не требуется введения дополнительного внутреннего или калибровочного пространства. Калибровочная симметрия означает здесь, что не существует никакого объективного 
свойства, которое могло бы отличить геометрию, определяемую связностью Вейля (1), от геометрии, определяемой эквивалентной ей связностью $\bar{\Gamma}$.

\section{3. УРАВНЕНИЯ КАЛИБРОВОЧНОГО ПОЛЯ}

Запишем калибровочно-инвариантный лагранжиан

$$
L=-\frac{1}{16} B_{i j k l} B^{i j k l}+\frac{1}{4} G_{i j k} S^{i j k}
$$

где $S^{i j k}$ - неизвестный пока ток - источник калибровочного поля, который должен иметь вид квадратичной функции от компонент величины, задающей спинорное представление калибровочной группы $G_{W}$. Вариационной процедурой получаем следующие уравнения калибровочного поля:

$$
\nabla_{i} B^{i j k l}+G_{i m}^{k} B^{i j m l}-G_{i m}^{l} B^{i j m k}+S^{j k l}=0 .
$$

Из (12) можно вывести уравнения для тока - источника калибровочного поля

$$
\nabla_{i} S^{i k l}+G_{i m}^{k} S^{i m l}-G_{i m}^{l} S^{i m k}=0 .
$$

Рассмотрим вектор тока

$$
Q^{j}=\frac{1}{2} v_{k l}\left(G_{i m}^{k} B^{i j m l}-G_{i m}^{l} B^{i j m k}+S^{j k l}\right) .
$$

Из уравнений поля следует, что ток сохраняется, если бивектор $v_{i j}$ удовлетворяет уравнениям $\nabla_{i} v_{j k}=0$. Однако соответствуюшая сохраняющаяся величина не является калибровочно-инвариантной. Такая же ситуация имеет место и в абстрактной теории калибровочных полей. Во всех предыдущих формулах предполагалось, что калибровочньй потенциал имеет размерность обратной длины. Чтобы явно выделить константу взаимодействия с калибровочным полем, нужно сделать подстановку

$$
G_{i k l} \Rightarrow \frac{\varepsilon}{\hbar c} G_{i k l}
$$

В пределе $\varepsilon \Rightarrow 0$ лагранжиан (11) переходит в чисто гравитационный

$$
L=-\frac{1}{16} R_{i j k l} R^{i j k l},
$$

который, как известно (см. [3, 4]), относится к перенормируемому типу.

Сравним калибровочную теорию Вейля с абстрактной теорией калибровочных полей. В последней рассматриваются произвольная полупростая группа Ли со структурными постоянными $f_{b c}^{a}$ и набор векторных полей. Пространственно-временные индексы поднимаются и опускаются с помощью метрического тензора $g_{i j}$, а параметрические индексы - с помошью групповой метрики (см. $[5,6]): g_{a b}=f_{a m}^{n} f_{b n}^{m}$. В нашем же случае метрический тензор одновременно является и групповым, а структурные постоянные не используются, так как для их введения потребовалось бы задать ортогональный базис в пространстве векторных полей, в чем нет необходимости. Такая возможность обусловлена тем, что для некоторых групп Ли, в том числе и для изучаемой, координаты на 
группе могут рассматриваться как тензорные поля в пространстве-времени, что приводит также к ситуации, когда отсутствует разделение между пространством-временем и калибровочным пространством.

\section{4. СПИНОРНОЕ ПРЕДСТАВЛЕНИЕ}

Рассмотрим вопрос о поле, которое является источником калибровочного поля Вейля и задает спинорное представление группы $G_{W}$. Введем 16 -компонентный объект, который можно определить как пространство всех ковариантных антисимметричных тензорных полей $f_{i_{1} \cdots i_{p}}(p$-ф̆орм, $p=0,1,2,3,4)$ на пространственно-временном многообразии с метрикой $g_{i j}$. В математической литературе принято употреблять также сокращенное название “дифференциальная форма”. Формой, следовательно, является величина

$$
F=\left(f, f_{i}, f_{i j}, f_{i j k}, f_{i j k l}\right) .
$$

Объекты такого рода впервые рассматривались в работе [7] (см. также [8-10]). Историю вопроса и дальнейшие ссылки можно найти в [11].

Поля типа (14) задают спинорное представление калибровочной группы Вейля. Чтобы доказать сформулированное утверждение, мы установим естественный лагранжиан для поля (14) и покажем, что он инвариантен относительно калибровочных преобразований, определяюших спинорное представление калибровочной группы $G_{W}$. Скалярную скобку двух полей типа (14) определим как

$$
(F, H)=\bar{f} h+\bar{f}_{i} h^{i}+\frac{1}{2 !} \bar{f}_{i j} h^{i j}+\frac{1}{3 !} \bar{f}_{i j k} h^{i j k}+\frac{1}{4 !} \bar{f}_{i j k l} h^{i j k l},
$$

где черта обозначает в данном случае комплексное сопряжение. Если $F$ есть форма, то обобщенный оператор ротора $d$ определяется следующим образом:

$$
d F=\left(0, \partial_{i} f, 2 \partial_{[i} f_{j]}, 3 \partial_{[i} f_{j k]}, 4 \partial_{[i} f_{j k l]}\right),
$$

где квадратные скобки обозначают альтернирование, $\partial_{i}=\partial / \partial x^{i}$. Простейший лагранжиан для поля $F$, который можно построить с помошью оператора $d$, имеет вид

$$
L_{d}(F)=(F, d F)+(d F, F)+m(F, F) .
$$

Заметим, что оператор внешнего дифференцирования (15) является единственным линейным оператором первого порядка, который коммутирует с преобразованиями группы диффеоморфизмов - группы симметрии гравитационных взаимодействий. С этой точки зрения лагранжиан (16) определяется однозначно. Если $\nabla_{i}-$ ковариантная производная по отношению к римановой связности метрики $g_{i j}$, определяемой соотношениями (2), то в формуле (15) частные производные можно заменить на ковариантные. Лагранжиан (16) не совсем удобен для исследования, так как оператор $d$ не является самосопряженным относительно скалярного произведения

$$
\langle F \mid H\rangle=\int(F, H) \sqrt{-g} d^{4} x .
$$

С помошью тождества

$$
\sum_{p=0}^{4} \frac{1}{p !} f_{k i_{1} \cdots i_{p}} h^{k i_{1} \cdots i_{p}}=\sum_{p=0}^{4} \frac{1}{p !}\left(p f_{i_{1} \cdots i_{p}}\right) h^{i_{1} \cdots i_{p}}
$$


нетрудно убедиться, что нужным свойством обладает оператор $\nabla=\delta+d$, где $\delta$ - оператор обобщенной дивергенции,

$$
\delta F=\left(-\nabla^{m} f_{m},-\nabla^{m} f_{m i},-\nabla^{m} f_{m i j},-\nabla^{m} f_{m i j k}, 0\right) .
$$

С помощью оператора $\nabla=\delta+d$ лагранжиан (16) запишется в виде

$$
L_{d}(F)=\frac{1}{2}(F, \nabla F)+\frac{1}{2}(\nabla F, F)+m(F, F)+\nabla_{i} T^{i},
$$

где

$$
T^{i}=\sum_{p=0}^{4} \frac{1}{p !} \bar{f}_{i_{1} \cdots i_{p}} f^{k i_{1} \cdots i_{p}}+\bar{f}^{k i_{1} \cdots i_{p}} f_{i_{1} \cdots i_{p}} .
$$

Таким образом, лагранжиан (16) эквивалентен лагранжиану

$$
L(F)=\frac{1}{2}(F, \nabla F)+\frac{1}{2}(\nabla F, F)+m(F, F),
$$

который и будет изучаться. Определим еше числовой оператор $\Lambda$, полагая

$$
\Lambda F=\left(f,-f_{i}, f_{i j},-f_{i j k}, f_{i j k l}\right) .
$$

Нетрудно убедиться в справедливости следующих соотношений:

$$
\Lambda^{2}=1, \quad \Lambda d+d \Lambda=0, \quad \nabla \Lambda+\Lambda \nabla=0 .
$$

Так как $\nabla d+d \nabla=\nabla^{2}$, то

$$
\nabla\left(\frac{1}{2} \nabla-d\right)+\left(\frac{1}{2} \nabla-d\right) \nabla=0 .
$$

Из (18) и (19) следует, что оператор

$$
\stackrel{*}{\nabla}=(\nabla-2 d) \Lambda
$$

коммутирует с оператором $\nabla$, а квадраты этих операторов равны:

$$
\nabla \stackrel{*}{\nabla}=\stackrel{*}{\nabla} \nabla, \quad \nabla^{2}=\stackrel{*}{\nabla}^{2} .
$$

Оператор $\stackrel{*}{\nabla}$ будем называть дуальным оператору $\nabla$, предполагая взаимность. Отмеченное свойство дуальности полей типа (14) дает возможность ввести важные алгебраические операторы, которые действуют в пространстве этих полей и задают спинорное представление группы $G_{W}$.

Делается это по аналогии с теорией Дирака следующим образом. Включим взаимодействие с электромагнитным полем. Для этого в соответствии с принципом "минимального электромагнитного взаимодействия" в операторах $\nabla$ и $\stackrel{*}{\nabla}$ сделаем подстановку

$$
\nabla_{i} \Rightarrow \nabla_{i}-\frac{i e}{\hbar c} A_{i}
$$


обозначим новые операторы через $D$ и $\stackrel{*}{D}$, соответственно. Найдем квадраты этих операторов:

$$
\stackrel{*}{D}^{2}=\nabla^{2}-\frac{i e}{\hbar c} \stackrel{*}{Q}\left(F_{i j}\right)+\frac{2 i e}{\hbar c} A^{i} \nabla_{i}+\frac{e^{2}}{\hbar^{2} c^{2}} A_{i} A^{i}+\frac{i e}{\hbar c} \nabla_{i} A^{i},
$$

где $F_{i j}$ - бивектор электромагнитного поля. Такого же вида формула получается и для дуального оператора $D$, только в ней вместо оператора $\stackrel{*}{Q}\left(F_{i j}\right)$ будет стоять дуальный ему оператор $Q\left(F_{i j}\right)$, их явный вид приводится ниже. Операторы $Q$ и $\stackrel{*}{Q}$, которые естественным образом появляются при возведении в квадрат операторов $D$ и $\stackrel{*}{D}$, определяются антисимметричными тензорными полями второго ранга (2-формами). Запишем явный вид операторов $Q\left(u_{i j}\right), \stackrel{*}{Q}\left(u_{i j}\right)$, заменяя бивектор электромагнитного поля произвольной 2-формой $u_{i j}$ :

$$
\begin{aligned}
\stackrel{*}{Q}\left(u_{i j}\right) F= & \left(\frac{1}{2} u^{m n} f_{m n}, \frac{1}{2} u^{m n} f_{m n i}+u_{m i} f^{m}, \frac{1}{2} u^{m n} f_{m n i j}+2 u_{m[i} f_{. j]}^{m}-u_{i j} f\right. \\
& \left.3 u_{m[i} f_{. j k]}^{m}-3 u_{[i j} f_{k]},-6 u_{[i j} f_{k l]}\right) \\
Q\left(u_{i j}\right) F= & \left(-\frac{1}{2} u^{m n} f_{m n},-\frac{1}{2} u^{m n} f_{m n i}+u_{m i} f^{m},-\frac{1}{2} u^{m n} f_{m n i j}+2 u_{m[i} f_{. j]}^{m}+u_{i j} f,\right. \\
& \left.3 u_{m[i} f_{. j k]}^{m}+3 u_{[i j} f_{k]}, 6 u_{[i j} f_{k l]}\right) .
\end{aligned}
$$

Можно показать, что операторы $Q\left(u_{i j}\right)$ и $\stackrel{*}{Q}\left(u_{i j}\right)$ коммутируют, что является проявлением свойства дуальности. Алгебра операторов

$$
J\left(u_{i j}\right)=\frac{1}{2} \stackrel{*}{Q}\left(u_{i j}\right)
$$

является замкнутой относительно обычной скобочной операции Ли. Действительно, согласно (21)

$$
\left[J\left(u_{i j}\right), J\left(v_{i j}\right)\right]=J\left(w_{i j}\right)
$$

где

$$
w_{i j}=u_{i m} v_{j .}^{m}-u_{j m} v_{i .}^{m},
$$

как и в рассмотренном ранее случае векторного поля. Из (23) следует, что операторы $J\left(u_{i j}\right)$ задают представление рассматриваемой калибровочной группы $G_{W}$ в пространстве полей (14). Так как

$$
\left(F, J\left(u_{i j}\right) H\right)=-\left(J\left(u_{i j}\right) F, H\right),
$$

то лагранжиан (17) будет инвариантным относительно калибровочных преобразований

$$
F \Rightarrow \bar{F}=\exp \left(J\left(u_{i j}\right)\right) F
$$

если

$$
\left[J\left(u_{i j}\right), \nabla\right]=0 .
$$


Соотношение (26) выполняется, если бивектор $u_{i j}$ удовлетворяет уравнениям

$$
\nabla_{i} u_{j k}=0
$$

Условия интегрируемости уравнений (27) следуют из тождеств Риччи и имеют вид $R_{i j k .}{ }^{m} u_{m l}+R_{i j l .}{ }^{m} u_{k m}=0$. Если $R_{i j l .}{ }^{m}=K\left(g_{i l} \delta_{j}^{m}-g_{j l} \delta_{i}^{m}\right)$, то уравнения (27) вообше не будут иметь решений. Таким образом, в пространствах постоянной кривизны лагранжиан (17) будет инвариантным относительно преобразований (25) только после введения калибровочного поля определенного типа. Какого именно, можно вывести следуюшим образом. Рассмотрим вариации вида $\delta F=J\left(u_{i j}\right) F$. На таком классе вариаций с точностью до производной Лагранжа имеем для лагранжиана (17)

$$
\delta L(F)=\frac{1}{4} \nabla_{i} u_{j k} S^{i j k}
$$

где $S^{i j k}$ - тензорное поле третьего ранга, антисимметричное по последним двум индексам,

$$
\begin{aligned}
S^{j k l}= & \sum_{p=0}^{4} \frac{1}{p !}\left(\bar{f}_{. i_{1} \cdots i_{p}}^{j} f^{k l i_{1} \cdots i_{p}}+2 g^{j[k} \bar{f}_{. i_{1} \cdots i_{p}}^{l]} f^{i_{1} \cdots i_{p}}-\right. \\
& \left.-2 \bar{f}^{i_{1} \cdots i_{p} j[k} f_{. i_{1} \cdots i_{p}}^{l]}-\bar{f}_{i_{1} \cdots i_{p}} f^{j k l i_{1} \cdots i_{p}}\right)+ \text { c. c. } .
\end{aligned}
$$

Таким образом, к лагранжиану (17) нужно добавить слагаемое вида

$$
L_{I}=\frac{1}{4} G_{j k l} S^{j k l}
$$

для того, чтобы обеспечить калибровочную инвариантность. Точно такое же слагаемое мы добавили к лагранжиану (11) калибровочного поля. Таким образом, явный вид тока - источника калибровочного поля - определяется калибровочной симметрией однозначно. Из (28) следует, что при преобразованиях $\delta F=J\left(u_{i j}\right) F$ тензор $S^{j k l}$ преобразуется по закону

$$
\delta S^{j k l}=u_{m}^{k} S^{i j m}-u_{m}^{j} S^{i k m} .
$$

Отсюда находим, что калибровочное поле $G_{i j k}$ преобразуется следуюшим образом:

$$
\delta G_{i j k}=\nabla_{i} u_{j k}+G_{i j m} u_{k .}^{m}-G_{i k m} u_{j .}^{m} .
$$

Согласно (7) поле $G_{i k l}$ является неабелевым калибровочным полем Вейля, а поле $F$, как показано, - его спинорным источником. В спинорном характере поля $F$ по отношению к группе $G_{W}$ можно убедиться непосредственно, сравнивая преобразования $(25)$ с преобразованиями (6). Пусть $a_{i}$ и $b_{i}$ - единичные ортогональные ковекторы, $(a, a)=$ $(b, b)=1,(a, b)=0$, где $(a, b)=a_{i} b_{j} g^{i j}$, и $u_{i j}=\alpha\left(a_{i} b_{j}-a_{j} b_{i}\right) \quad(u=\alpha a \wedge b)$. Если $R v^{i}=\left(a_{j} b^{i}-a^{i} b_{j}\right) v^{j}$, то после некоторых вычислений получаем

$$
\begin{aligned}
\exp (\alpha R) v^{i}= & v^{i}-a^{i}(a, v)-b^{i}(b, v)+\left(\cos \alpha a^{i}+\sin \alpha b^{i}\right)(a, v)+ \\
& +\left(\cos \alpha b^{i}-\sin \alpha a^{i}\right)(b, v) .
\end{aligned}
$$


Для оператора $J\left(u_{i j}\right)$ имеем $J^{2}=-\alpha^{2} / 4$, и, следовательно,

$$
\exp (\alpha J(a \wedge b))=\cos \frac{\alpha}{2}+\sin \frac{\alpha}{2} J(a \wedge b) .
$$

Полагая теперь $\alpha=0,2 \pi$, нетрудно убедиться, что векторные поля задают тензорное представление калибровочной группы, а поля типа (14) - ее спинорное представление. Теория поля $G_{i j k}$ сформулирована вьше, а в следуюшем разделе мы остановимся на связи меж ду неабелевым калибровочным потенциалом Вейля и кручением Картана. На существование такой связи указывает хотя бы то, что оба поля являются тензорными полями одного и того же типа и оба определяют метрические связности.

\section{5. КРУЧЕНИЕ И КАЛИБРОВОЧНАЯ СИММЕТРИЯ}

В настоящее время открытое Картаном кручение находится в центре многочисленных исследований, ставяших целью установить физический смысл кручения и связь между общей теорией относительности и физикой микромира. Здесь мы рассмотрим этот вопрос в рамках неабелевой калибровочной теории Вейля. Пусть дана некоторая линейная связность и $\Gamma_{j k}^{i}-$ компоненты или символы Кристоффеля этой связности. Тогда, как впервые было показано Картаном [12], линейная связность однозначно определяет тензорное поле $K_{j k}^{i}=(1 / 2)\left(\Gamma_{j k}^{i}-\Gamma_{k j}^{i}\right)$, которое называется тензором кручения. Геометрия Римана-Картана задается метрикой и тензором кручения линейной связности, согласованной с рассматриваемой метрикой. Этим связность, называемая метрической, определяется однозначно, так как

$$
\Gamma_{j k}^{i}=\left\{\begin{array}{c}
i \\
j k
\end{array}\right\}+K_{j k}^{i}+g^{i l} K_{l j}^{m} g_{m k}+g^{i l} K_{l k}^{m} g_{m j} .
$$

Из (1) следует, что тензор кручения заданной связности Вейля равен

$$
K_{j k}^{i}=\frac{1}{2}\left(G_{j k}^{i}-G_{k j}^{i}\right)
$$

или

$$
K_{i j k}=\frac{1}{2}\left(G_{i j k}-G_{j i k}\right),
$$

где $K_{i j k}=K_{i j}^{l} g_{l k}$. Отсюда находим, что

$$
G_{i j l}=K_{i j l}-K_{i l j}-K_{j l i} .
$$

Подобно (30) для связности Вейля $\bar{\Gamma}_{j k}^{i}=\left\{\begin{array}{l}i \\ j k\end{array}\right\}+\bar{G}_{j k}^{i}$, эквивалентной (1), мы имеем

$$
\bar{K}_{i j k}=\frac{1}{2}\left(\bar{G}_{i j k}-\bar{G}_{j i k}\right) .
$$

Учитывая взаимно однозначное соответствие между тензором кручения и неабелевым калибровочным полем Вейля, вообше говоря, можно поставить вопрос о связи между тензорами $\bar{K}_{i j k}$ и $K_{i j k}$, коль скоро согласно (8)

$$
\bar{G}_{i j k}=G_{i l m} T_{j}^{l} T_{k}^{m}+g_{l m} T_{j}^{l} \nabla_{i} T_{k}^{m} .
$$


Однако получающиеся при этом соотношения не являются ни простыми, ни естественными. Действительно, если положить

$$
G_{i j k}=\frac{1}{2}\left(G_{i j l}-G_{j i l}\right)+\frac{1}{2}\left(G_{i j l}+G_{j i l}\right)=K_{i j l}+N_{i j l}
$$

где $N_{i j l}=K_{l i j}+K_{l j i}$, то нетрудно видеть, что в закон преобразования тензора кручения при калибровках будет входить тензор $N_{i j k}$ и, соответственно, в закон преобразования тензора $N_{i j k}$ при калибровках будет входить тензор кручения. Это вполне естественно, так как тензор $G_{i j k}$ кососимметричен по второму и третьему индексам, в то время как тензор кручения кососимметричен по первым двум индексам. Поэтому в соотношении (31) перепутываются индекс, участвующий в калибровочном преобразовании (8), и индекс, не затрагиваемый таковым. Следовательно, с точки зрения калибровочной симметрии естественным геометрическим объектом является тензор $G_{i j k}$, определяюший конгруэнтный перенос. Именно для этого тензора записаны калибровочно-инвариантные уравнения (12), которые определяются калибровочной симметрией фактически однозначно. Сейчас легко понять, почему в литературе для тензора кручения с равным успехом вводятся и исследуются всевозможные лагранжианы. Произвол обусловлен тем, что, как показано, принципы симметрии трудно применить к тензору кручения самому по себе, и только введение калибровочного поля Вейля делает этот вопрос простым.

В заключение этого раздела отметим связь между калибровочными преобразованиями и римановой геометрией. Второе слагаемое в правой части соотношения (8) обрашается в нуль, если $\nabla_{i} T_{k}^{j}=0$. В абстрактной теории калибровочных полей это соответствует переходу от локальных преобразований к глобальным. В рассматриваемом случае уравнения $\nabla_{i} T_{k}^{j}=0$ вообше могут не иметь нетривиальных решений, например, когда $g_{i j}$ является метрикой пространства постоянной кривизны. Заметим также, что геометрические соотношения, как и физические законы, не зависят ни от выбора системы координат, ни от выбора базиса в изучаемых векторных пространствах, так что все соотношения, которые установлены выше, могут быть записаны в любой системе координат и в любом базисе, в том числе и ортогональном.

\section{6. УРАВНЕНИЯ ПОЛЯ}

В этом разделе будет показано, что калибровочно-инвариантные уравнения поля (14) в определенной калибровке могут быть записаны с помошью ковариантной производной по отношению к связности (1) и, следовательно, в соответствии с (30) и (31) как уравнения в пространстве Римана-Картана. Возьмем калибровочно-инвариантный лагранжиан

$$
L=L(F)+\frac{1}{4} G_{i j k} S^{i j k}
$$

и найдем его вариацию по отношению к $F$. В результате получаем следуюшее соотношение:

$$
\begin{aligned}
\delta L= & \delta L(F)+\frac{1}{2} \sum_{p=0}^{4} \frac{1}{p !} \delta \bar{f}^{i_{1} \cdots i_{p}}\left(G^{m} f_{m i_{1} \cdots i_{p}}-p G_{\left[i_{1}\right.} f_{\left.i_{2} \cdots i_{p}\right]}+\right. \\
& +\frac{p}{2} D_{m n\left[i_{1}\right.} f_{\left.\ldots i_{2} \cdots i_{p}\right]}^{m n}+\frac{p(p-1)}{2} D_{\left[i_{1} i_{2}|m|\right.} f_{\left.i_{3} \cdots i_{p}\right]}^{m}- \\
& \left.-\frac{1}{3 !} P^{j k l} f_{j k l i_{1} \cdots i_{p}}-\frac{1}{3 !} p(p-1)(p-2) P_{\left[i_{1} i_{2} i_{3}\right.} f_{\left.i_{4} \cdots i_{p}\right]}\right)+ \text { c. c. }
\end{aligned}
$$


где

$$
G_{m}=g^{j k} G_{j k m}, \quad P_{i j k}=3 G_{[i j k]}, \quad D_{i j k}=-P_{i j k}+2 G_{i j k} .
$$

K индексу, выделенному вертикальными черточками, операция альтернирования не относится. Из (34) следуют калибровочно-инвариантные уравнения поля $F$. Запись этих уравнений сушественно упрошается в определенной калибровке, которую можно вывести следуюшим образом. В лагранжиане $L(F)$ ковариантную производную $\nabla_{i}$ по отношению к римановой связности метрики $g_{i j}$ заменим на ковариантную производную $\nabla_{i}^{*}$ по отношению к связности (1). Такой подстановкой мы также вводим калибровочное поле в лагранжиан (17). Новый лагранжиан обозначим как $L_{G}(F)$. Можно показать, что лагранжиан $L_{G}(F)$ совпадает с лагранжианом $(33)$, если поле $G_{i j k}$ удовлетворяет уравнениям

$$
G_{i j k}+G_{j k i}+G_{k i j}=0 .
$$

Вариационной процедурой получаем из лагранжиана $L_{G}(F)$ следующие уравнения поля:

$$
-\widehat{\nabla}^{l} f_{l i_{1} \cdots i_{p}}+p \widehat{\nabla}_{\left[i_{1}\right.} f_{\left.i_{2} \cdots i_{p}\right]}+m f_{i_{1} \cdots i_{p}}=0
$$

где

$$
\widehat{\nabla}_{i}=\nabla_{i}^{*}-\frac{1}{2} G_{i}, \quad p=0,1,2,3,4 .
$$

Эти уравнения совпадают с калибровочно-инвариантными уравнениями для поля (14) в калибровке (35). При варьировании лагранжиана $L_{G}(F)$ нужно учесть, что

$$
\stackrel{*}{\nabla}_{i} A^{i}=\frac{1}{\sqrt{-g}} \partial_{i}\left(\sqrt{-g} A^{i}\right)+G_{i} A^{i}
$$

где $g$ - определитель метрического тензора. Учитывая связь между тензором кручения и рассматриваемым неабелевым калибровочным полем, можно также сказать, что уравнения поля $F$, получаемые варьированием лагранжиана $(33)$, совпадают с уравнениями этого поля в пространстве-времени Римана-Картана при условии, что тензор кручения удовлетворяет уравнениям

$$
K_{i j k}+K_{j k i}+K_{k i j}=0
$$

\section{7. ЗАКЛЮЧЕНИЕ}

Суммируем результаты проведенного рассмотрения и остановимся на некоторых вопpocax. Приведенная здесь интерпретация конгруэнтного переноса позволяет выявить связь между классической дифференциальной геометрией и теорией калибровочных полей. Важно подчеркнуть еше раз принципиальную значимость этой связи, которая состоит в том, что в рассматриваемом случае не требуется введения абстрактного калибровочного пространства. Установленные соотношения между калибровочным полем Вейля и тензором кручения Картана позволяют по-новому рассматривать физическую интерпретацию кручения в рамках калибровочного принципа. Существование спинорного источника у калибровочного поля Вейля является интересной особенностью этого поля. В связи с этим возникает вопрос о том, где могло бы проявиться рассмотренное взаимодействие. В пространстве-времени Минковского уравнения (27) являются вполне интегрируемыми и поэтому введение неабелева калибровочного поля хотя и возможно, но в известном смысле оно не вытекает из первых принципов. Таким образом, 
калибровочную симметрию, определяемую группой $G_{W}$, естественно рассматривать в этом случае как глобальную внутреннюю симметрию. Поэтому более перспективной представляется следующая возможность. Как уже отмечалось, уравнение (27) не имеет решений в пространствах постоянной кривизны. В этом случае введение калибровочного поля Вейля является в определенном смысле необходимым хотя бы потому, что глобальной группы внутренней симметрии просто нет. Интересным пространственно-временным многообразием указанного типа является пространство-время де Ситтера, которое с физической точки зрения обычно рассматривается как космологическая модель. Таким образом, вейлевское взаимодействие могло бы проявиться в космологических масштабах. Конечно, этим ни в коей мере не закрывается область малых масштабов. Обшее же замечание состоит в том, что все вопросы, обсуждаемые в литературе в связи с попытками физической интерпретации кручения, могут быть исследованы с точки зрения неабелевой калибровочной теории Вейля.

\section{Список литературы}

[1] Б. М. Барбашов, А. Б. Пестов. ТМФ. 1995. Т. 104. С. 429.

[2] H. Weyl. Space-Time-Matter. New York: Dover Publications: INC, 1922.

[3] K. S. Stelle. Phys. Rev. D. 1977. V. 16. P. 953.

[4] Б. Л. Воронов, И. В. Тютин. ЯФ. 1984. Т. 39(4). С. 998.

[5] $P$. Утияма. Инвариантная теория взаимодействий. В кн.: Элементарные частицы и компенсирующие поля. Ред. Д. Иваненко. М.: Мир, 1964. С. 250-273.

[6] А.А. Славнов, Л.Д. Фаддеев. Введение в квантовую теорию калибровочных полей. М.: Наука, 1988

[7] D. Ivanenko, L. Landau. Zeitschrift fur Physik. 1928. Bd. 48. S. 340.

[8] П. К. Рашевский. УМН. 1955. Т. 10. С. 1.

[9] А. Б. Пестов. ТМФ. 1978. Т. 34. С. 48.

[10] В. А. Желнорович. Теория спиноров и ее приложения в физике и механике. М.: Наука, 1982.

[11] D. D. Ivanenko, Yu. N. Obukhov. Ann. der Phys. 1985. V. 42. P. 59.

[12] É. Cartan. Comptes Rendus de 1'Academie des Sciences (Paris). 1922. V. 174. P. 593.

Поступила в редакцию 29.I.1997 г., после доработки 21.IV.1997 г.

\section{B. M. Barbashov, A. B. Pestov \\ ANTISYMMETRIC TENSOR FIELDS AND THE WEYL GAUGE THEORY}

A spinor current source is found in the Weyl non-Abelian gauge theory that does not contain an abstract gauge space. It is shown that the corresponding spinor representation of the Weyl gauge group can be constructed in the space of antisymmetric tensor fields and as a 16-component quantity for which a gauge-invariant Lagrangian is determined. The connection between the Weyl gauge potential and the Cartan torsion, field and the problem of the possible manifestation of the considered interactions, are discussed. 